\title{
CLASSIFICATION OF SMOOTH SCHUBERT VARIETIES IN THE SYMPLECTIC GRASSMANNIANS
}

\author{
JAEHYUN HONG
}

\begin{abstract}
A Schubert variety in a rational homogeneous variety $G / P$ is defined by the closure of an orbit of a Borel subgroup $B$ of $G$. In general, Schubert varieties are singular, and it is an old problem to determine which Schubert varieties are smooth. In this paper, we classify all smooth Schubert varieties in the symplectic Grassmannians.
\end{abstract}

\section{Introduction}

A rational homogeneous manifold $S=G / P$ is a projective manifold, where a connected complex semisimple group $G$ acts transitively. Under the action of a Borel subgroup $B$ of $G, S$ has finitely many orbits. The closure of a $B$ orbit in $S$ is called a Schubert variety of $S$. In general, Schubert varieties are singular, and it is an old problem to determine which Schubert varieties are smooth. Lakshmibai-Weyman and Brion-Polo have studied the singular loci of Schubert varieties of $S$, when $S$ is a compact Hermitian symmetric space ([9] and [2]). In particular, they showed that in this case any smooth Schubert variety in $S$ is a homogeneous submanifold of $S$ associated to a subdiagram of the marked Dynkin diagram of $S$. For example, a Schubert variety of the Grassmannian $\operatorname{Gr}(k, V)$ of $k$-subspaces in a vector space $V$ is smooth if and only if it is a linearly embedded sub-Grassmannian.

More generally, when $S$ is associated to a long simple root, we have:

Theorem 1.1 (Proposition 3.7 of Hong-Mok [4]). Let $S=G / P$ be a rational homogeneous manifold associated to a long simple root. Then any smooth Schubert variety in $S$ is a homogeneous submanifold of $S$ associated to a subdiagram of the marked Dynkin diagram of $S$.

On the other hand, when $S$ is associated to a short simple root, there is a smooth Schubert variety that is not homogeneous. Let $V$ be a vector space with a symplectic form $\omega$, i.e., a nondegenerate skew-symmetric bilinear form.

Received March 8, 2015.

2010 Mathematics Subject Classification. Primary 14Mxx, 53Cxx.

Key words and phrases. Schubert varieties, symplectic Grassmannians. 
It follows from the nondegeneracy of the skew-symmetric form that $V$ is evendimensional. The variety $G r_{\omega}(k, V)$ of isotropic $k$-subspaces in $V$ is called a symplectic Grassmannian. It is a rational homogeneous manifold under the action of the symplectic group $S p(V)$ associated to a short simple root. Similarly, for an odd-dimensional vector space $W$ with a skew-symmetric form $\psi$ of maximal rank, the variety $G r_{\psi}(k, W)$ of isotropic $k$-subspaces in $W$ is called an odd symplectic Grassmannian (Mihai [10]). It is smooth, but is no longer homogeneous. When $S$ is the symplectic Grassmannian $G r_{\omega}(k, V)$, take a hyperplane $W$ of $V$ such that the rank of $\omega$ when restricted to $W$ is maximal. Then the odd symplectic Grassmannian $G r_{\omega}(k, W)$ is a Schubert variety of $S$, which is smooth and is not homogeneous.

Another interesting smooth Schubert variety of the symplectic Grassmannian $G r_{\omega}(k, V)$ is a linear space contained in $G r_{\omega}(k, V)$. Here, we consider $G r_{\omega}(k, V)$ as a subvariety of $\mathbb{P}\left(\bigwedge^{k} V\right)$. Take an isotropic $(k-1)$-subspace $F$ of $V$ and a subspace $F^{\prime}$ of $V$. Then the variety $G r_{\omega}\left(k, V ; F, F^{\prime}\right)$ of isotropic $k$-subspaces of $V$ containing $F$ and contained in $F^{\prime}$ is a Schubert variety of $G r_{\omega}(k, V)$, and is a linear space contained in $G r_{\omega}(k, V)$, because any onedimensional subspace of $V$ is isotropic. However, $G r_{\omega}(k, V ; F, V)$ is not associated to a subdiagram of the marked diagram of $G r_{\omega}(k, V)$, even though it is homogeneous under its automorphism group.

In this paper we will classify smooth Schubert varieties in the symplectic Grassmannian $G r_{\omega}(k, V)$ and prove that these are all smooth Schubert varieties of $G r_{\omega}(k, V)$.

Theorem 1.2. Let $S=G / P$ be the symplectic $\operatorname{Grassmannian} \operatorname{Gr}_{\omega}(k, V)$. Then a smooth Schubert variety of $S$ is either a homogeneous submanifold associated to a subdiagram of the marked diagram of $S$, an odd symplectic Grassmannian, or a linear space.

There are many results on smoothness and singularities of Schubert varieties. For various smoothness criteria and applications, see Billey-Lakshmibai [1]. It could be possible to get classification of smooth Schubert varieties of the symplectic Grasssmannian $G r_{\omega}(k, V)$ by using their method. But, even in the case that it is possible to do, we still need some combinatorial work to interpret it geometrically and to derive results like Theorem 1.1 or Theorem 1.2.

In this paper, we will apply a more geometric method to classify smooth Schubert varieties: parallel transport of varieties of minimal rational tangents along minimal rational curves (Section 3.2 of Hong-Mok [3] and Proposition 3.2 of [4]). A new ingredient is the study on the closures of orbits of a Borel subgroup of $L$ in a horospherical $L$-variety, which can be considered as a generalization of Schubert varieties in rational homogeneous manifolds.

In Section 2, we review basic definitions on Schubert varieties and the varieties of minimal rational tangents. In Section 3, we study Schubert varieties in the symplectic Grassmannian $G r_{\omega}(k, V)$ (Proposition 3.1). In Section 4, we investigate the varieties of minimal rational tangents of smooth Schubert 
varieties (Proposition 4.1), and classify candidates of the varieties of minimal rational tangents of smooth Schubert varieties in $G r_{\omega}(k, V)$ (Proposition 4.2). From this we obtain the classification of smooth Schubert varieties in $G r_{\omega}(k, V)$ (Proposition 4.7).

\section{Preliminary}

\subsection{Schubert varieties}

Let $G$ be a connected complex simple group. Take a Borel subgroup $B$ of $G$ and a maximal torus $T$ in $B$. Denote by $\Delta^{+}$the set of positive roots. Then $\Delta:=\Delta^{+} \cup-\Delta^{+}$is the set of all roots. Let $\mathcal{S}=\left\{\alpha_{1}, \ldots, \alpha_{\ell}\right\}$ be the set of simple roots. For a root $\beta=\sum_{i=1}^{\ell} n_{i} \alpha_{i}$, denote by $n_{j}(\beta)$ the coefficient in $\alpha_{j}$ of $\beta$, so that $\beta=\sum_{i=1}^{\ell} n_{i}(\beta) \alpha_{i}$.

Let $\mathfrak{t}$ be the Lie algebra of $T$. To each simple root $\alpha_{k}$ we associate a parabolic subgroup $P$ of $G$, whose Lie algebra $\mathfrak{p}$ is given by $\mathfrak{p}=\mathfrak{t}+\sum_{n_{k}(\alpha) \geq 0} \mathfrak{g}_{\alpha}$. The reductive part of $\mathfrak{p}$ is given by $\mathfrak{t}+\sum_{n_{k}(\alpha)=0} \mathfrak{g}_{\alpha}$, and the nilpotent part $\mathfrak{u}_{P}$ of $\mathfrak{p}$ is given by $\sum_{n_{k}(\alpha)>0} \mathfrak{g}_{\alpha}$. The homogeneous manifold $S=G / P$ is called the rational homogeneous manifold associated to the simple root $\alpha_{k}$. We will use the notation $\left(G, \alpha_{k}\right)$ for $S$, and $G$ will be often given by its type.

Let $\mathcal{W}$ be the Weyl group of $G$ with respect to the maximal torus $T$ in $B$. For each $w \in \mathcal{W}$, define $\Delta(w)=\left\{\beta \in \Delta^{+}: w(\beta) \in-\Delta^{+}\right\}$. Define a subset $\mathcal{W}^{P}$ of $\mathcal{W}$ by

$$
\mathcal{W}^{P}:=\left\{w \in \mathcal{W}: \Delta(w) \subset \Delta\left(\mathfrak{u}_{P}\right)\right\},
$$

where $\Delta\left(\mathfrak{u}_{P}\right)=\left\{\alpha \in \Delta^{+}: n_{\alpha_{k}}(\alpha)>0\right\}$. Then we have the cell decomposition

$$
S=\coprod_{w \in \mathcal{W}^{P}} B \cdot e_{w},
$$

where $e_{w}=w P$, for $w \in \mathcal{W}^{P}$, are $T$-fixed points in $S$. For each $w \in \mathcal{W}^{P}$, the $B$-orbit $B . e_{w}$ is isomorphic to a cell $\mathbb{C}^{\ell(w)}$ of dimension equal to the length $\ell(w)$ of $w$ in $\mathcal{W}$. The closure $S(w)$ of B.e $e_{w}$ is called the Schubert variety of type $w$. We call the point $e_{w}$ the base point of $S(w)$.

\subsection{Rationally saturated subvarieties}

Let $(X, L)$ be a polarized uniruled projective manifold, where $L$ is an ample line bundle on $X$. Fix a minimal rational component $\mathcal{K}$ and the variety of minimal rational tangents $\mathcal{C}(X) \subset \mathbb{P}(T X)$.

For a projective manifold $X \subset \mathbb{P}(V)$ that is uniruled by lines contained in $X$, the variety $\mathcal{K}$ of lines lying on $X$ is a minimal rational component, and the variety $\mathcal{K}_{x}$ of lines in $X$ passing through $x \in X$ is isomorphic to the variety $\mathcal{C}_{x}(X) \subset \mathbb{P}\left(T_{x} X\right)$ of tangent directions to lines in $X$ passing through $x \in X$. The union $\mathcal{C}(X)=\cup_{x \in X} \mathcal{C}_{x}(X)$ is the variety of minimal rational tangents of $X$. 
When we speak of the variety of minimal rational tangents of a rational homogeneous manifold $S$ associated to a simple root, we will assume that $S$ is equipped with the minimal rational component $\mathcal{K}$, consisting of lines $\mathbb{P}^{1}$ contained in $S$ after we embed $S$ into $\mathbb{P}^{N}$ by the ample generator of the Picard group of $S$. For more details on the variety of minimal rational tangents and references, see Section 3.1 of [4].

An irreducible subvariety $Z$ of $X$ is said to be rationally saturated if

(1) $\mathbb{P}\left(T_{z} Z\right) \cap \mathcal{C}_{z}(X) \neq \emptyset$ for a smooth point $z \in Z$, and

(2) for a general smooth point $z \in Z$ and for a general minimal rational curve $C$ on $X$ passing through $z, C$ must lie on $Z$ whenever $C$ is tangent to $Z$ at $z$.

Then the family of minimal rational curves contained in $Z$ can be considered as a minimal rational component of $Z$, with respect to which the variety $\mathcal{C}_{z}(Z)$ of minimal rational tangents of $Z$ at $z \in Z$ is equal to $\mathbb{P}\left(T_{z} Z\right) \cap \mathcal{C}_{z}(X)$.

Proposition 2.1. Let $X \subset \mathbb{P}(V)$ be a projective submanifold uniruled by lines in $\mathbb{P}(V)$ contained in $X$, equipped with the minimal rational component of $X$ consisting of lines lying on $X$. Let $Z$ be an irreducible linear section of $X$. Assume that $\mathbb{P}\left(T_{z} Z\right) \cap \mathcal{C}_{z}(X) \neq \emptyset$ for a general smooth point $z$ of $Z$. Then $Z$ is rationally saturated.

Proof. If $Z=X \cap \mathbb{P}(W)$, then any line $C$ through a smooth point of $Z$ tangent to $Z$ is tangent to $\mathbb{P}(W)$, and thus is contained in $\mathbb{P}(W)$. Therefore, $C$ is contained in $Z=X \cap \mathbb{P}(W)$.

Proposition 2.2. A Schubert variety $S_{0}$ of a rational homogeneous manifold $S$ is rationally saturated.

Proof. Let $S_{0}$ be a Schubert variety of a rational homogeneous manifold $S$. Then $S_{0}$ is irreducible and is a linear section of $S$. Furthermore, a Schubert variety of dimension one, which is a line, is contained in $S_{0}$ and can be translated by an element in the Weyl group $\mathcal{W}$ to have a non-trivial intersection with the open $B$-orbit in $S_{0}$ (see the proof of Proposition 3.1 of [4]). Hence, there always exists a line passing through a general smooth point of $S_{0}$. By Proposition 2.1, $S_{0}$ is rationally saturated.

Remark. When $S_{0}$ is not smooth, it may happen that a general line through $e_{w}$ intersects the singular locus of $S_{0}$.

\section{Schubert varieties of the symplectic Grassmannians}

Let $V$ be a complex vector space of dimension $2 n$ equipped with a nondegenerate skew-symmetric bilinear form $\omega$. Take a basis $\left\{e_{1}, \ldots, e_{2 n}\right\}$ of $V$, such that $\omega\left(e_{n-i}, e_{n+i+1}\right)=-\omega\left(e_{n+i+1}, e_{n-i}\right)=1$ for $1 \leq i \leq n$, and all other $\omega\left(e_{i}, e_{j}\right)$ are zero. Define $F_{j} \subset V$ by the subspace generated by $e_{1}, \ldots, e_{j}$ for $1 \leq j \leq 2 n$. Then, $F_{n-i}^{\perp}=F_{n+i}$ for $1 \leq i \leq n$, and we obtain an isotropic flag $F_{\bullet}: 0 \subsetneq F_{1} \subsetneq \cdots \subsetneq F_{2 n}=V$. The subgroup of $G=S p(V)$ consisting 
of elements fixing this flag is a Borel subgroup $B$ of $G$. Let $P$ be the isotropy group of $G$ at $\left[F_{k}\right]$. Then, $G r_{\omega}(k, V)=G / P$, and $B$ is contained in $P$. As a rational homogeneous manifold associated to a simple root, $G r_{\omega}(k, V)$ has type $\left(C_{n}, \alpha_{k}\right)$.

A multi-index $I=\left(1 \leq i_{1}<i_{2}<\cdots<i_{k} \leq 2 n\right)$ is said to be admissible if, for each $1 \leq i \leq n, I$ contains at most one of $i$ or $2 n+1-i$. For an admissible $I$, define

$$
\begin{aligned}
& C_{I}:=\left\{E \in G r(k, V): \operatorname{dim}\left(E \cap F_{i_{\alpha}}\right)=\alpha, i_{\alpha} \leq i<i_{\alpha+1}, 1 \leq \alpha \leq k\right\}, \\
& X_{I}:=\left\{E \in G r(k, V): \operatorname{dim}\left(E \cap F_{i_{\alpha}}\right) \geq \alpha, 1 \leq \alpha \leq k\right\},
\end{aligned}
$$

where we put $i_{k+1}=2 n+1$. Then, $C_{I}$ is an orbit of the Borel subgroup $B$, and $X_{I}$ is a Schubert variety of $G r_{\omega}(k, V)$ (Section 2.5 of [10]). Let $E_{I}$ be the subspace of $V$ generated by $e_{i_{1}}, \ldots, e_{i_{k}}$. Then, $\left[E_{I}\right]$ is the base point of the Schubert variety $X_{I}$.

Proposition 3.1. For $0 \leq a<k<b \leq 2 n-a$, define

$$
G r_{\omega}\left(k, V ; F_{a}, F_{b}\right):=\left\{E \in G r_{\omega}(k, V): F_{a} \subset E \subset F_{b}\right\} .
$$

Then

(1) $\operatorname{Gr}_{\omega}\left(k, V ; F_{a}, F_{b}\right)$ is a Schubert variety of $G r_{\omega}(k, V)$ if $b-a \leq n-a$ or $(n-a)+(k-a) \leq b-a \leq 2 n-2 a$.

(2) $\operatorname{Gr}_{\omega}\left(k, V ; F_{a}, F_{b}\right)$ is a homogeneous submanifold associated to a subdiagram if $k<b \leq n$ or $b=2 n-a$, is an odd symplectic Grassmannian if $b=2 n-a-1$, and is a linear space if $a=k-1$.

(3) $\operatorname{Gr}_{\omega}\left(k, V ; F_{a}, F_{b}\right)$ is singular if $a \leq k-2$ and $n+k-a \leq b \leq 2 n-a-2$.

Proof. (1) Note that $G r_{\omega}\left(k, V ; F_{a}, F_{b}\right)$ is isomorphic to $G r_{\widetilde{\omega}}\left(k-a, \widetilde{V} ; 0, F_{b} / F_{a}\right)$, where $\widetilde{V}=F_{a}^{\perp} / F_{a}$ is of dimension $2 n-2 a$ and $\widetilde{\omega}$ is the induced symplectic form on $\widetilde{V}$. Thus, we may assume that $a=0$.

If $b \leq n$ or $n+k \leq b$, then $G r_{\omega}\left(k, V ; 0, F_{b}\right)$ is equal to

$$
\left\{E \in G r_{\omega}(k, V): \operatorname{dim}\left(E \cap F_{b-k+1}\right) \geq 1, \ldots, \operatorname{dim}\left(E \cap F_{b}\right) \geq k\right\} .
$$

Therefore, $G r_{\omega}\left(k, V ; 0, F_{b}\right)$ is a Schubert variety.

(2) It is easy to check.

(3) Put $S_{1}:=G r_{\omega}\left(k, V ; F_{a}, F_{b}\right)$. Assume that $a=0$. The proof will be similar for $a \neq 0$. For $[E] \in S_{1}$,

$$
T_{[E]}\left(S_{1}\right)=\left\{\varphi \in E^{*} \otimes F_{b} / E: \omega\left(e, \varphi\left(e^{\prime}\right)\right)+\omega\left(\varphi(e), e^{\prime}\right)=0, \forall e, e^{\prime} \in E\right\} .
$$

Now, set

$$
\begin{aligned}
D_{[E]}^{1}\left(S_{1}\right):= & E^{*} \otimes\left(F_{b} \cap E^{\perp}\right) / E, \\
D_{[E]}^{2}\left(S_{1}\right):= & \left\{\varphi \in E^{*} \otimes\left(F_{b} /\left(F_{b} \cap E^{\perp}\right)\right): \omega\left(e, \varphi\left(e^{\prime}\right)\right)+\omega\left(\varphi(e), e^{\prime}\right)=0,\right. \\
& \left.\forall e, e^{\prime} \in E\right\} .
\end{aligned}
$$

Then

$$
0 \rightarrow D_{[E]}^{1}\left(g S_{1}\right) \rightarrow T_{[E]}\left(g S_{1}\right) \rightarrow D_{[E]}^{2}\left(g S_{1}\right) \rightarrow 0
$$


For $E_{0}=\left\langle e_{b-k+1}, \ldots, e_{b}\right\rangle, F_{b} \cap E_{0}^{\perp}$ has dimension $b-k$, and $F_{b} /\left(F_{b} \cap E_{0}^{\perp}\right) \simeq E_{0}^{*}$ via $\omega$. Thus, $\operatorname{dim} T_{[E]}\left(S_{1}\right)=k(b-2 k)+\frac{1}{2} k(k+1)=k(b-k)-\frac{1}{2} k(k-1)$. We will show that for $E=F_{k}=\left\langle e_{1}, \ldots, e_{k}\right\rangle$, $\operatorname{dim} T_{[E]}\left(S_{1}\right)>\operatorname{dim} T_{\left[E_{0}\right]}\left(S_{1}\right)$, which implies that $S_{1}$ is singular.

If $k \leq 2 n-b$, then $E^{\perp} \supseteqq F_{b}$. Therefore, $T_{[E]}\left(S_{1}\right)=E^{*} \otimes\left(F_{b} / E\right)$ has dimension $k(b-k)>\operatorname{dim} S_{1}$. If $k>2 n-b$, then $E^{\perp} \subsetneq F_{b}$. Thus, $D_{[E]}^{1}\left(S_{1}\right)=$ $E^{*} \otimes\left(E^{\perp} / E\right)$ has dimension $k(2 n-2 k)$, and $D_{[E]}^{2}\left(S_{1}\right) \simeq S^{2}\left(F_{b} / E^{\perp}\right)$ has dimension $\frac{1}{2}(b-2 n+k)(b-2 n+k+1)$. Therefore,

$$
\begin{aligned}
\operatorname{dim} T_{[E]}\left(S_{1}\right) & =k(2 n-2 k)+\frac{1}{2}(b-2 n+k)(b-2 n+k+1) \\
& =k(b-k)-k(b-2 n+k)+\frac{1}{2}(b-2 n+k)(b-2 n+k+1) \\
& =k(b-k)+\frac{1}{2}(b-2 n+k)(b-2 n-k+1) \\
& =k(b-k)-\frac{1}{2} k(k-1)+\frac{1}{2}(2 n-b)(2 n-b-1) \\
& >\operatorname{dim} S_{1} \text { if } b<2 n-1 .
\end{aligned}
$$

Remark. If $n-a<b-a<(n-a)+(k-a)$, then $G r_{\omega}\left(k, V ; F_{a}, F_{b}\right)$ is not irreducible, and thus is not a schubert variety. For example, $G r_{\omega}\left(3, \mathbb{C}^{10} ; F_{0}, F_{7}\right)$ is the union $X_{\{3,6,7\}} \cup X_{\{3,5,7\}}$ of two Schubert varieties.

\section{Classification}

\subsection{Varieties of minimal rational tangents of smooth Schubert vari- eties}

Let $S=G / P$ be a rational homogeneous manifold associated to a simple root. Then, the Fano variety $F_{1}(S)$ of lines on $S$ has at most two $G$-orbits, or equivalently, the variety $\mathcal{C}_{x}(S)$ of minimal rational tangents of $S$ at $x$ has at most two orbits under the action of the isotropy group $P_{x}$ of $G$ at $x$ (see the proof of Proposition 4.1(3)). We call a line corresponding to a point in the open $G$-orbit in $F_{1}(S)$ a general line. Let $\mathcal{C}_{x}(S)^{\text {gen }}$ denote the subvariety of $\mathcal{C}_{x}(S)$ consisting of the tangent directions of general lines in $S$.

Let $S_{1}$ be a Schubert variety of $S$. Then the stabilizer $\operatorname{Stab}_{G}\left(S_{1}\right)$ of $S_{1}$ in $G$ is a parabolic subgroup of $G$. We define a general point in $S_{1}$ as a point $x$ in the open orbit of $\operatorname{Stab}_{G}\left(S_{1}\right)$ in $S_{1}$. In particular, the base point of $S_{1}$ is a general point. We define a general point of $\mathcal{C}_{x}\left(S_{1}\right)$ as a point in $\mathcal{C}_{x}\left(S_{1}\right) \cap \mathcal{C}_{x}(S)^{\text {gen }}$.

The following necessary conditions for the smoothness of a Schubert variety will be used to classify smooth Schubert varieties in the symplectic Grassmannian.

Proposition 4.1. Let $S=G / P$ be a rational homogeneous manifold associated to a simple root, and let $S_{1}$ be a smooth Schubert variety. In addition, let $x$ be 
a general point of $S_{1}$, and $L_{x}$ be the reductive part of the isotropy group of $G$ at $x$. Then:

(0) $S_{1}$ is uniruled by lines of $S$ lying on $S_{1}$ and is of Picard number one.

(1) The variety $\mathcal{C}_{x}\left(S_{1}\right)$ of minimal rational tangents of $S_{1}$ at $x$ is a smooth linear section $\mathcal{C}_{x}(S) \cap \mathbb{P}\left(T_{x} S_{1}\right)$.

(2) The variety $\mathcal{C}_{x}(S)$ of minimal rational tangents of $S$ at $x$ has an open dense orbit under the action of $L_{x}$, and has finitely many orbits under the action of a Borel subgroup of $L_{x}$.

(3) $\mathcal{C}_{x}\left(S_{1}\right)$ is the closure of an orbit of a Borel subgroup of $L_{x}$ in $\mathcal{C}_{x}(S)$.

The properties (0), (1), and (3) from Proposition 4.1 for the variety $\mathcal{C}_{x}\left(S_{1}\right)$ of minimal rational tangents of a smooth Schubert variety $S_{1}$ are used to classify smooth Schubert varieties in a rational homogeneous manifold associated to a long simple root (see the proof of Proposition 3.7 of [4]). We will prove that $\mathcal{C}_{x}\left(S_{1}\right)$ has the same properties when $S$ is associated to a short simple root.

Proof. Let $S=G / P$ be a rational homogeneous manifold associated to a simple root, and let $S_{1}$ be a smooth Schubert variety. Let $x$ be a general point of $S_{1}$, and $L_{x}$ be the reductive part of the isotropy group of $G$ at $x$.

(0) follows from Proposition 3.1 of [4].

(1) follows from Proposition 3.1 of [4].

(2) If $S$ is associated to a long simple root, then $\mathcal{C}_{x}(S)$ is again a rational homogeneous manifold under the action of $L_{x}$ (see the description of $\mathcal{C}_{x}(S)$ on p. 342 of [4]). If $S$ is associated to a short simple root, then $\mathcal{C}_{x}(S)$ is not a rational homogeneous manifold under the action of $L_{x}$. We recall the description of the variety of minimal rational tangents of $S$ when $S$ is associated to a short simple root (Hwang-Mok [7], [8]):

(a) If $S$ is of type $\left(C_{n}, \alpha_{k}\right)$, then $L_{x}$ is $S L\left(E^{*}\right) \times S p(Q)$, and $\mathcal{C}_{x}(S)$ is the projectivization of the cone

$$
\left\{u \otimes q+c u^{2}: u \in E^{*}, q \in Q, c \in \mathbb{C}\right\} \backslash\{0\}
$$

in $\left(E^{*} \otimes Q\right) \oplus S^{2} E^{*}$, where $E^{*}$ is a vector space of dimension $k$ and $Q$ is a vector space of dimension $2 m=2(n-k)$ with a symplectic form.

(b) If $S$ is of type $\left(F_{4}, \alpha_{3}\right)$, then $L_{x}$ is $S L(W) \times S L(E)$, and $\mathcal{C}_{x}(S)$ is the projectivization of the cone

$$
\left\{v^{*} \otimes e+w \otimes e^{2}:\left\langle v^{*}, w\right\rangle=0, v^{*} \in W^{*}, v \in W, e \in E\right\} \backslash\{0\}
$$

in $\left(W^{*} \otimes E\right) \oplus\left(W \otimes S^{2} E\right)$, where $W$ is a complex vector space of dimension 3 and $E$ is a complex vector space of dimension 2 .

(c) If $S$ is of type $\left(F_{4}, \alpha_{4}\right)$, then $L_{x}$ is $B_{3}(=\operatorname{Spin}(7))$, and $\mathcal{C}_{x}(S)$ is the projectivization of the closure of the $B_{3}$-orbit of $v_{3}+v_{1}$ in $V_{B_{3}}\left(\omega_{3}\right) \oplus$ $V_{B_{3}}\left(\omega_{1}\right)$, where $v_{3}$ is a highest weight vector of the spinor representation $V_{B_{3}}\left(\omega_{3}\right)$ of $B_{3}$ (of dimension 8 ), and $v_{1}$ is a highest weight vector of the standard representation $V_{B_{3}}\left(\omega_{1}\right)$ of $B_{3}$ (of dimension 7 ). 
Moreover, $\mathcal{C}_{x}(S)$ has an open dense orbit $\Omega$ under the action of $L_{x}$, which is defined as follows:

(a) If $S$ is of type $\left(C_{n}, \alpha_{k}\right)$, then $\Omega$ is the projectivization of the cone

$$
\left\{u \otimes q+c u^{2}: u \neq 0 \in E^{*}, q \neq 0 \in Q, c \neq 0 \in \mathbb{C}\right\}
$$

in $\left(E^{*} \otimes Q\right) \oplus S^{2} E^{*}$.

(b) If $S$ is of type $\left(F_{4}, \alpha_{3}\right)$, then $\Omega$ is the projectivization of the cone

$$
\begin{aligned}
& \left\{v^{*} \otimes e+w \otimes e^{2}:\left\langle v^{*}, w\right\rangle=0, v^{*} \neq 0 \in W^{*}, w \neq 0 \in W, e \neq 0 \in E\right\} \\
& \quad \text { in }\left(W^{*} \otimes E\right) \oplus\left(W \otimes S^{2} E\right) .
\end{aligned}
$$

(c) If $S$ is of type $\left(F_{4}, \alpha_{4}\right)$, then $\Omega$ is the complement in $\mathcal{C}_{x}(S)$ of the union of the $B_{3}$-orbit of $\left[v_{3}\right]$ in $\mathbb{P}\left(V_{B_{3}}\left(\omega_{3}\right)\right)$ and the $B_{3}$-orbit of $\left[v_{1}\right]$ in $\mathbb{P}\left(V_{B_{3}}\left(\omega_{1}\right)\right)$.

We will prove this again for later use in the case when $S$ is of type $\left(C_{n}, \alpha_{k}\right)$, i.e., when $S$ is the symplectic Grassmannian. The proof will be similar for other cases.

Consider the projection map

$$
\pi: \Omega \longrightarrow \mathbb{P}\left(\left(E^{*} \otimes Q\right) \otimes S^{2} E^{*}\right)
$$

defined by $\left[u \otimes q+c u^{2}\right] \in \Omega \stackrel{\pi}{\longmapsto}\left[(u \otimes q) \otimes\left(c u^{2}\right)\right]=\left[(u \otimes q) \otimes\left(u^{2}\right)\right] \in \mathbb{P}\left(\left(E^{*} \otimes\right.\right.$ $\left.Q) \otimes S^{2} E^{*}\right)$, where $u \neq 0 \in E^{*}, q \neq 0 \in Q, c \neq 0 \in \mathbb{C}$. Then, the image $\pi(\Omega)$ is $\mathbb{P}\left(E^{*}\right) \times \mathbb{P}(Q)$, which is a rational homogeneous manifold under the action of $L_{x}$ embedded into $\mathbb{P}\left(\left(E^{*} \otimes Q\right) \otimes S^{2} E^{*}\right)$ (the Segre embedding of the product of $\left(\mathbb{P}\left(E^{*}\right) \subset \mathbb{P}\left(S^{3} E^{*}\right)\right)$ and $\left.\mathbb{P}(Q)\right)$. Furthermore, the isotropy group $H_{1}$ at a point $\left[u \otimes q+c u^{2}\right]$ is contained in the isotropy group $H_{2}$ at $\left[(u \otimes q) \otimes\left(u^{2}\right)\right]$, and the quotient $H_{2} / H_{1} \simeq \mathbb{C}^{\times}:=\mathbb{C} \backslash\{0\}$ acts transitively on the fiber over $\left[(u \otimes q) \otimes\left(u^{2}\right)\right]$. Therefore, $\Omega$ is homogeneous under the action of $L_{x}$.

Let $w$ be the element in $\mathcal{W}^{P}$ corresponding to $S_{1}$ (See Section 2.1). Let $L$ be the reductive part of $P$. We may take $e_{w}$ as a general point $x$ in $S_{1}$, and it follows that the isotropy group $L_{x}$ at $x$ is $w(L)$. Therefore, $w(B \cap L)$ is a Borel subgroup of $L_{x}$. Since $\pi(\Omega)=\mathbb{P}\left(E^{*}\right) \times \mathbb{P}(Q)$ has finitely many orbits under the action of $w(L \cap B)$, so does $\Omega$.

(3) follows from (1) and (2) (see the proof of Proposition 3.7 of [4]). We will repeat the arguments for convenience.

Let $w$ be the element in $\mathcal{W}^{P}$ corresponding to $S_{1}$, as in the proof of (2). Since $w \in \mathcal{W}^{P}$, we have that $\Delta(\omega) \subset \Delta\left(\mathfrak{u}_{P}\right)$ and so $w(B \cap L)$ is contained in $B \cap w(L)$. Therefore, $w(B \cap L)$ is a Borel subgroup of $L_{x}$, and is contained in $B \cap L_{x}$.

Since $B$ acts on $S_{1}$ invariantly, $B \cap L_{x}$ acts on $\mathcal{C}_{x}\left(S_{1}\right)$ invariantly. Hence, the Borel subgroup $w(B \cap L)$ of $L_{x}$ acts on $\mathcal{C}_{x}\left(S_{1}\right)$ invariantly. By $(2), \mathcal{C}_{x}(S)$ has only finitely many orbits under the action of $w(B \cap L)$, and it follows from (1) that $\mathcal{C}_{x}\left(S_{1}\right)$ is smooth and thus is irreducible. Hence, $\mathcal{C}_{x}\left(S_{1}\right)$ has a unique open orbit under the action of the Borel subgroup $w(B \cap L)$ of $L_{x}$. Consequently, $\mathcal{C}_{x}\left(S_{1}\right)$ is the closure of the Borel subgroup $w(B \cap L)$ of $L_{x}$. 
Remark. If $S$ is a rational homogeneous manifold associated to a long simple root, and $S_{1}$ is a smooth Schubert variety of $S$, then $\mathcal{C}_{x}(S)$ is a product of rational homogeneous manifolds associated to long simple roots, and $\mathcal{C}_{x}\left(S_{1}\right)$ is a smooth Schubert variety of $\mathcal{C}_{x}(S)$. Therefore, we may use induction to classify smooth Schubert varieties in $S$ (see the proof of Proposition 3.7 in [4]).

If $S$ is associated to a short simple root, then $\mathcal{C}_{x}(S)$ is no longer a rational homogeneous manifold under the action of $L_{x}$. However, it has an open orbit isomorphic to a $\mathbb{C}^{\times}$-bundle over a rational homogeneous manifold, which still has finitely many orbits under the action of a Borel subgroup of $L_{x}$ (Proposition $4.1(2))$.

In general, for a reductive group $L$, a normal $L$-variety that has only finitely many orbits under the action of a Borel subgroup of $L$ is said to be spherical. As in the case of rational homogeneous manifolds, a spherical $L$-variety also has a cell-decomposition by the orbits of a Borel subgroup of $L$. It is not easy to determine the smoothness of their closures in general. We will compute these closures in $\mathcal{C}_{x}(S)$ when $S$ is the symplectic Grassmannian.

\subsection{Characterization of smooth Schubert varieties}

Recall that $\left\{F_{\bullet}\right\}=\left\{F_{0}=0 \subset F_{1} \subset \cdots \subset F_{2 n}=V\right\}$ is an isotropic flag of $(V, \omega)$, and $B$ is the Borel subgroup of $G=S p(V)$ fixing the flag $\left\{F_{\bullet}\right\}$. Let $P$ be the isotropy group of $G$ at the base point $[E]:=\left[F_{k}\right]$, and let $L$ be the reductive part of the isotropy group $P$. Then the flag $\left\{F_{\bullet}\right\}$ of $V$ induces a flag of $E^{*}$ and of $E^{\perp} / E$ :

$$
\begin{aligned}
& \left\{0 \subset\left(E /\left(F_{k-1} \cap E\right)\right)^{*} \subset \cdots \subset\left(E /\left(F_{1} \cap E\right)\right)^{*} \subset E^{*}\right\}, \\
& \left\{0 \subset\left(F_{k+1} \cap E^{\perp}\right) / E \subset \cdots \subset\left(F_{2 n-k-1} \cap E^{\perp}\right) / E \subset E^{\perp} / E\right\} .
\end{aligned}
$$

Let $\mathcal{X}_{1, a}$ be the Schubert variety $\mathbb{P}\left(\left(E /\left(F_{a} \cap E\right)\right)^{*}\right)$ of $\mathbb{P}\left(E^{*}\right)$, where $0 \leq a \leq$ $k-1$, and let $\mathcal{X}_{2, b}$ be the Schubert variety $\mathbb{P}\left(\left(F_{b} \cap E^{\perp}\right) / E\right)$ of $\mathbb{P}\left(E^{\perp} / E\right)$, where $k+1 \leq b \leq 2 n-k$. Then $\mathcal{X}_{1, a} \times \mathcal{X}_{2, b}$ are Schubert varieties of $\mathbb{P}\left(E^{*}\right) \times \mathbb{P}\left(E^{\perp} / E\right)$. Let $\overline{\mathcal{X}}_{1, a}^{o}\left(\mathcal{X}_{2, b}^{o}\right.$, respectively) denote the open cell of $\mathcal{X}_{1, a}\left(\mathcal{X}_{2, b}\right.$, respectively $)$. Then, $\pi^{-1}\left(\mathcal{X}_{1, a}^{o} \times \mathcal{X}_{2, b}^{o}\right)$ is an $(L \cap B)$-orbit in the open $L$-orbit $\Omega \subset \mathcal{C}_{[E]}(S)$, where $\pi: \Omega \rightarrow \mathbb{P}\left(E^{*}\right) \times \mathbb{P}\left(E^{\perp} / E\right)$ is the projection map in Proposition 4.1(2). Let $\mathcal{Z}_{a, b}$ be the closure of $\pi^{-1}\left(\mathcal{X}_{1, a}^{o} \times \mathcal{X}_{2, b}^{o}\right)$ in $\mathcal{C}_{[E]}(S)$. Then, by Proposition $4.1(2)$, we get the following.

Proposition 4.2. Let $S$ be the symplectic Grassmannian $G r_{\omega}(k, V)$. Let $P$ be the isotropy group of $G=S p(V)$ at the base point $[E]$, and let $L$ be the reductive part of $l P$. Then the closures of $L \cap B$-orbits in $\mathcal{C}_{[E]}(S)$ are the following:

$$
\mathcal{X}_{1, a} \times \mathcal{X}_{2, b}, \mathcal{Z}_{a, b}, \mathcal{X}_{1, a}
$$

where $0 \leq a \leq k-1, k+1 \leq b \leq 2 n-k$.

Here, we consider $\mathcal{X}_{1, a} \times \mathcal{X}_{2, b}$ as a subvariety of $\mathbb{P}\left(E^{*} \otimes\left(E^{\perp} / E\right)\right) \cap \mathbb{C}_{[E]}(S)=$ $\mathbb{P}\left(E^{*}\right) \times \mathbb{P}\left(E^{\perp} / E\right)$ and $\mathcal{X}_{1, a}$ as a subvariety of $\mathbb{P}\left(S^{2}\left(E^{*}\right)\right) \cap \mathcal{C}_{[E]}(S)=\mathbb{P}\left(E^{*}\right)$. Then, $\mathcal{Z}_{a, b}$ intersects $\mathbb{P}\left(E^{*} \otimes\left(E^{\perp} / E\right)\right)$ in $\mathcal{X}_{1, a} \times \mathcal{X}_{2, b}$, and intersects $\mathbb{P}\left(S^{2}\left(E^{*}\right)\right)$ in 
$\mathcal{X}_{1, a}$. We define a general point of $\mathcal{Z}_{a, b}$ as a point in the complement $\mathcal{Z}_{a, b} \backslash \mathcal{X}_{1, a} \times$ $\mathcal{X}_{2, b}$ of $\mathcal{X}_{1, a} \times \mathcal{X}_{2, b}$

Proposition 4.3. Let $S$ be the symplectic Grassmannian $G r_{\omega}(k, V)$, and let $[E]$ be the base point of $S$. For $0 \leq a<k$ and $(k-a<b-a \leq n-a$, or $(n-a)+(k-a) \leq b-a \leq 2 n-2 a)$, set $S_{a, b}:=G r_{\omega}\left(k, V ; F_{a}, F_{b}\right)$ and let $\left[E_{a, b}\right]$ be the base point of $S_{a, b}$. Then

$\mathcal{C}_{\left[E_{a, b}\right]}(S) \cap \mathbb{P}\left(T_{\left[E_{a, b}\right]} S_{a, b}\right)=\left\{\begin{array}{l}\mathcal{X}_{1, a} \times \mathcal{X}_{2, b} \quad \text { if } k-a<b-a \leq n-a, \\ \mathcal{Z}_{a, b} \quad \text { if }(n-a)+(k-a) \leq b-a \leq 2 n-2 a,\end{array}\right.$

after we identify $T_{\left[E_{a, b}\right]} S$ with $T_{[E]} S$ via $w \in \mathcal{W}^{P}$, with $w([E])=\left[E_{a, b}\right]$.

Note that, by Proposition 3.1, $S_{a, b}=G r_{\omega}\left(k, V ; F_{a}, F_{b}\right)$ is a Schubert variety for $a, b$ as given in Proposition 4.3.

Proposition 4.4. Let $S$ be the symplectic Grassmannian $G r_{\omega}(k, V)$. Let $[E]$ be the base point of $S$ and let $P$ be the isotropy group at $[E]$. Assume that $0 \leq a<k$ and $(n-a)+(k-a) \leq b-a \leq 2 n-2 a$. Then, at a general point $\alpha$ of $\mathcal{Z}_{a, b}$ and for any $h \in P$ sufficiently close to the identity element $e \in P$ and satisfying $T_{\alpha}\left(h \mathcal{Z}_{a, b}\right)=T_{\alpha}\left(\mathcal{Z}_{a, b}\right)$, we have $h \mathcal{Z}_{a, b}=\mathcal{Z}_{a, b}$.

Proof. The proof is similar to the proof of Proposition 4.3 in [4]. We may assume that $a=0$. Let $n+k \leq b \leq 2 n$. Then, $F_{b} /\left(F_{b} \cap E^{\perp}\right)$ is isomorphic to $E^{*}$, via the symplectic form $\omega$. For a general $\alpha=e^{*} \otimes v$ in $\mathcal{Z}_{a, b}=\mathbb{P}\left\{e^{*} \otimes v \in\right.$ $\left.E^{*} \otimes F_{b} / E: \omega(v, \cdot)=\lambda e^{*}\right\}$, the tangent space $T_{\alpha}\left(\mathcal{Z}_{a, b}\right)$ is given by

$$
e^{*} \otimes\left(F_{b} \cap E^{\perp}\right) / E+\left\{f^{*} \otimes v+e^{*} \otimes v_{f}: f \in E\right\},
$$

where for $f \in E, v_{f}$ is an element in $F_{b}$ such that $\omega\left(v_{f}, \cdot\right)=f^{*}$ on $E$, and thus determines $F_{b}$.

Proposition 4.5. Let $S$ be the symplectic Grassmannian $G r_{\omega}(k, V)$ and let $S_{a, b}$ be the Schubert variety of the form $G r_{\omega}\left(k, V ; F_{a}, F_{b}\right)$, where $0 \leq a \leq k-2$ and $(n-a)+(k-a) \leq b-a \leq 2 n-2 a$. Let $x$ be the base point $\left[E_{a, b}\right]$ of $S_{a, b}$. Then the following properties hold:

(1) Let $Z$ be a smooth Schubert variety (with respect to a Borel subgroup $\tilde{B}$ of $G$ which may not be equal to $B$ ). If $Z$ contains $x$ as a general point with $\mathcal{C}_{x}(Z)=\mathcal{C}_{x}\left(S_{a, b}\right)$, then $Z$ is equal to $S_{a, b}$.

(2) There does not exist such $a Z$ as in (1) if $0 \leq a \leq k-2$ and $(n-a)+$ $(k-a) \leq b-a \leq 2 n-2 a-2$.

Before giving the proof, let us roughly outline how it works. We will use similar arguments as those in the proofs of Proposition 3.2 and Proposition 3.7 in [4]. There, we assumed the following two properties for the variety $\mathcal{Z}=\mathcal{C}_{x}\left(S_{0}\right)$ of minimal rational tangent of the 'model' Schubert variety $S_{0}$ :

(I) At a general point $\alpha \in \mathcal{Z}$, for any $h \in P_{x}$ sufficiently close to the identity element $e \in P_{x}$ and satisfying $T_{\alpha}(h \mathcal{Z})=T_{\alpha}(\mathcal{Z})$, we must have $h \mathcal{Z}=\mathcal{Z}$. 
(II) Any local deformation of $\mathcal{Z}$ in $\mathcal{C}_{x}(S)$ is induced by the action of $P_{x}$.

The same argument works for $S_{0}=S_{a, b}$ when $0 \leq a \leq k-2$ and $2 n-2 a-1 \leq$ $b-a \leq 2 n-2 a$. However, it does not work for $S_{0}=S_{a, b}$ when $0 \leq a \leq k-2$ and $(n-a)+(k-a) \leq b-a \leq 2 n-2 a-2$. There are two differences. One is that the property (II) no longer holds. The other is that our 'model' Schubert variety $S_{a, b}$ is not smooth (Proposition 3.1(3)). We will overcome these difficulties by assuming that $Z$ is not just a smooth subvariety of $S$ uniruled by lines, but also is a Schubert variety. Then we will use the property that $S_{a, b}$ has a line not intersecting the singular locus of $S_{a, b}$.

Lemma 4.6. Let $S_{a, b}$ be the Schubert variety $G r_{\omega}\left(k, V ; F_{a}, F_{b}\right)$, where $0 \leq$ $a \leq k-2$ and $(n-a)+(k-a) \leq b-a \leq 2 n-2 a$. Then we have a sequence of locally closed submanifolds $\mathcal{V}^{0}=\{x\} \subsetneq \mathcal{V}^{1} \subsetneq \cdots \subsetneq \mathcal{V}^{m}$ of $S_{a, b}$ such that $\operatorname{dim} \mathcal{V}^{m}=\operatorname{dim} S_{a, b}$, and any point in $\mathcal{V}^{k}$ can be connected to a point in $\mathcal{V}^{k-1}$ by a line.

Proof. This follows from the fact that $S_{a, b}$ is a smooth uniruled projective variety of Picard number one when $0 \leq a \leq k-2$ and $2 n-2 a-1 \leq b-a \leq 2 n-2 a$ (see Section 4.3 of [6]). The same arguments as in Section 4.3 of [6] work if there is a line in $S_{a, b}$ contained in the smooth locus of $S_{a, b}$.

We may assume that $a=0$. Let $S_{a, b}^{\prime}:=\left\{E \in S_{a, b}: F_{2 n-b} \cap E=0\right\}$. Then, $S_{a, b}^{\prime}$ is contained in the smooth locus of $S_{a, b}$, and the line $\left\{E \in S_{a, b}\right.$ : $\left.\left\langle e_{n-k+1}, \ldots, e_{n-1}\right\rangle \subset E \subset\left\langle e_{n-k+1}, \ldots, e_{n-1}, e_{n}, e_{n+1}\right\rangle\right\}$ is contained in $S_{a, b}^{\prime}$.

Proof of Proposition 4.5. (1) Let $Z$ be a smooth Schubert variety of $S$ (with respect to a Borel subgroup $\tilde{B}$ of $G$ which may not be equal to $B$ ). Then, $Z$ is uniruled by lines, and of Picard number one (Proposition 4.1(0)). Assume that $Z$ contains $x$ as a general point, with $\mathcal{C}_{x}(Z)=\mathcal{C}_{x}\left(S_{a, b}\right)$. Then the locus of lines in $S_{a, b}$ passing through $x$ is contained in $Z$.

Take a general line $C$ through $x$ contained in $S_{a, b}$. Let $C^{\prime}$ be the intersection of $C$ with the orbit $S_{a, b}^{g e n}$ of $x$ under the action of the stabilizer $\operatorname{Stab}_{G}\left(S_{a, b}\right)$ of $S_{a, b}$ in $G$. Since $x$ is a general point of $S_{a, b}, C^{\prime}$ is a Zariski open subset of $C$. Similarly, let $C^{\prime \prime}$ be the intersection of $C$ with the orbit $Z^{\text {gen }}$ of $x$ under the action of the stabilizer $\operatorname{Stab}_{G}(Z)$ of $Z$ in $G$. Then $C^{\prime \prime}$ is also a Zariski open subset of $C$.

Let $y \in C^{\prime} \cap C^{\prime \prime}$. Then, since $y$ is a general point of $S_{a, b}, \mathcal{C}_{x}\left(S_{a, b}\right) \subset$ $\mathbb{P}\left(T_{x}\left(S_{a, b}\right)\right)$ is projectively equivalent to $\mathcal{C}_{y}\left(S_{a, b}\right) \subset \mathbb{P}\left(T_{y}\left(S_{a, b}\right)\right)$. Similarly, since $y$ is a general point of $Z, \mathcal{C}_{x}(Z) \subset \mathbb{P}\left(T_{x} Z\right)$ is projectively equivalent to $\mathcal{C}_{y}(Z) \subset$ $\mathbb{P}\left(T_{y} Z\right)$. Hence, $\mathcal{C}_{y}\left(S_{a, b}\right) \subset \mathbb{P}\left(T_{y}\left(S_{a, b}\right)\right)$ is projectively equivalent to $\mathcal{C}_{y}(Z) \subset$ $\mathbb{P}\left(T_{y} Z\right)$. By Proposition 3.4 of [5], there exists $h \in P_{y}$ such that $h \mathcal{C}_{y}\left(S_{a, b}\right)=$ $\mathcal{C}_{y}(Z)$. (In [5] we consider the case when $b=2 n-a$. The same arguments work in other cases, too.) By Lemma 2.8 of [3] we have that $T_{\alpha}\left(h \mathcal{C}_{y}\left(S_{a, b}\right)\right)=$ $T_{\alpha}\left(\mathcal{C}_{y}(Z)\right)$, and by Proposition 4.4 we have that $\mathcal{C}_{y}\left(S_{a, b}\right)=\mathcal{C}_{y}(Z)$. Therefore, 
the locus of lines in $S_{a, b}$ passing through $y$ is contained in $Z$. Repeating the same arguments and using Lemma 4.6, we get that $S_{a, b}$ is contained in $Z$.

If $\operatorname{dim} S_{a, b}<\operatorname{dim} Z$, then by the same arguments as in the proof of Proposition 3.7 of [4] we have an integrable distribution $\mathcal{W}$ of $Z$ with compact leaves $g S_{a, b}$, where $g$ lies in some subvariety $G^{\prime}$ of $G$, such that for a general point $g \in G^{\prime}$ and for a general point $z \in g S_{a, b}$, any line in $Z$ passing through $z$ lies in $g S_{a, b}$. By arguments from the same proof again, the existence of such a $\mathcal{W}$ contradicts the fact that $Z$ is a uniruled projective manifold of Picard number one. Therefore, we have $\operatorname{dim} S_{a, b}=\operatorname{dim} Z$, from which it follows that $Z$ is equal to $S_{a, b}$.

(2) follows from (1) and Proposition 3.1(3).

Now, we will show that there is no smooth Schubert variety of the form $G r_{\omega}\left(k, V ; F_{a}, F_{b}\right)$ other than those given in Proposition 3.1.

Proposition 4.7. Smooth Schubert varieties of the symplectic Grassmannian $G r_{\omega}(k, V)$ are of the form $G r_{\omega}\left(k, V ; F_{a}, F_{b}\right)$, where one of the following holds:

(1) $0 \leq a<k$ and $(k<b \leq n$ or $b=2 n-a)$,

(2) $0 \leq a<k$ and $b=2 n-a-1$,

(3) $a=k-1$ and $n+1 \leq b \leq 2 n-1$.

Recall that a line in $S=G r_{\omega}(k, V)$ is general if it corresponds to a point in the open orbit of $G$ in $F_{1}(S)$, that a point in $\mathcal{C}_{[E]}(S) \cap \mathbb{P}\left(E^{*} \otimes\left(E^{\perp} / E\right)\right.$ corresponds to a non-generic line passing through $[E]$, and that a point in its complement corresponds to a generic line passing through $[E]$. We describe how they differ by comparing them with lines in the Grassmannian $G r(k, V)$.

Let $\left(E_{k-1}, E_{k+1}\right)$ be a pair of a $(k-1)$-subspace $E_{k-1}$ of $V$ and a $(k+1)$ subspace $E_{k+1}$ of $V$, such that $E_{k-1} \subset E_{k+1}$. Then,

$$
L_{E_{k-1}, E_{k+1}}:=\left\{[E]: E_{k-1} \subset E \subset E_{k+1}\right\}
$$

is a line in $\mathbb{P}\left(\wedge^{k} V\right)$ contained in $\operatorname{Gr}(k, V)$, and any line in $\operatorname{Gr}(k, V)$ is obtained in this way.

The line $L_{E_{k-1}, E_{k+1}}$ is contained in the symplectic Grassmannian $G r_{\omega}(k, V)$ if $E_{k-1}$ is isotropic and $E_{k+1}$ is a subspace of $E_{k-1}^{\perp}$. Moreover, if $E_{k+1}$ is isotropic (not isotropic, respectively), then $L_{E_{k-1}, E_{k+1}}$ is non-generic (generic, respectively).

Proof of Proposition 4.7. Let $S_{1}$ be a smooth Schubert variety of $S$. In addition, let $w$ be the corresponding element $w \in \mathcal{W}^{P}$ and let $[E]$ be the base point of $S_{1}$. By Proposition $4.1(1), \mathcal{C}_{[E]}\left(S_{1}\right)$ is the linear section $\mathcal{C}_{[E]}(S) \cap \mathbb{P}\left(T_{[E]} S_{1}\right)$ of $\mathcal{C}_{[E]}(S)$. By Proposition $4.1(3)$ and its proof, $\mathcal{C}_{[E]}\left(S_{1}\right)$ is the closure of the Borel subgroup $w(L \cap B)$ of $L_{[E]}$.

Assume that $S_{1}$ does not contain a generic line. Then, $\mathcal{C}_{[E]}\left(S_{1}\right)$ is contained in $\mathbb{P}\left(E^{*} \otimes\left(E^{\perp} / E\right)\right) \cap \mathcal{C}_{[E]}(S)$. By Proposition $4.2, \mathcal{C}_{[E]}\left(S_{1}\right)$ is $\mathcal{X}_{1, a} \times \mathcal{X}_{2, b}$ for some $a, b$. 
Consider $S_{1}$ as a subvariety of the Grassmannian $G r(k, V)$. Then, by Proposition 3.5 of [4] and the fact that $S_{1}$ has Picard number one, $S_{1}$ is a subgrassmannian $\operatorname{Gr}\left(k, V ; F_{a}, F_{b}\right)$. However, $\operatorname{Gr}\left(k, V ; F_{a}, F_{b}\right)$ is not contained in $G r_{\omega}(k, V)$ unless $F_{b}$ is an isotropic subspace of $V$, i.e., $b \leq n$. Therefore, $S_{1}$ is of the form $\operatorname{Gr}\left(k, V ; F_{a}, F_{b}\right)$ for some $b \leq n$ and $\mathcal{C}_{[E]}\left(S_{1}\right)$ is of the form $\mathcal{X}_{1, a} \times \mathcal{X}_{2, b}$ for some $b \leq n$.

Assume that $S_{1}$ contains a generic line. Then, $S_{1}$ contains a non-generic line too, because $S_{1}$ is compact. By Proposition 4.2, $\mathcal{C}_{[E]}\left(S_{1}\right)$ is $\mathcal{Z}_{a, b}$ for some $a$ and $b$. Therefore, $\mathcal{Z}_{a, b}=\mathcal{C}_{[E]}(S) \cap \mathbb{P}\left(T_{[E]} S_{1}\right)$, and the linear span $\left\langle\mathcal{Z}_{a, b}\right\rangle$ of $\mathcal{Z}_{a, b}$ is contained in $\mathbb{P}\left(T_{[E]} S_{1}\right)$. In fact, $\left\langle\mathcal{Z}_{a, b}\right\rangle$ is equal to $\mathbb{P}\left(T_{[E]} S_{1}\right)$, because $\mathcal{C}_{[E]}(S)$ is nondegenerate in $\mathbb{P}\left(T_{[E]} S\right)$. The linear span $\left\langle\mathcal{Z}_{a, b}\right\rangle$ of $\mathcal{Z}_{a, b}$ is $\mathbb{P}\left(\left(\left(E / F_{a} \cap\right.\right.\right.$ $\left.\left.E)^{*} \otimes\left(\left(F_{b} \cap E^{\perp}\right) / E\right)\right) \oplus\left(S^{2}\left(E / F_{a} \cap E\right)^{*}\right)\right)$ when we identify $T_{[E]} S$ with $\left(E^{*} \otimes\right.$ $\left.\left(E^{\perp} / E\right)\right) \oplus\left(S^{2} E^{*}\right)$. Thus, $\mathbb{P}\left(T_{[E]} S_{1}\right)=\mathbb{P}\left(\left(\left(E / F_{a} \cap E\right)^{*} \otimes\left(\left(F_{b} \cap E^{\perp}\right) / E\right)\right) \oplus\right.$ $\left.\left(S^{2}\left(E / F_{a} \cap E\right)^{*}\right)\right)$.

Assume that $a=0$ (the proof for the case when $a \geq 1$ will be similar). Consequently, we have $\mathbb{P}\left(T_{[E]} S_{1}\right)=\mathbb{P}\left(\left(E^{*} \otimes\left(\left(F_{b} \cap E^{\perp}\right) / E\right)\right) \oplus\left(S^{2} E^{*}\right)\right)$. We claim that if $k \neq 1$, it follows that $b \geq n+k$. One can prove this by exploiting the Schubert cells in $G r_{\omega}(k, V)$, or by using the fact that $G r_{\omega}\left(k, V ; 0, F_{b}\right)$ is not a Schubert variety unless $b \geq n+k$. We leave the proof to the reader. Therefore, by Proposition 4.5(2), we have that $b=2 n-1$ or $2 n$.

Now, Theorem 1.2 follows from Proposition 3.1 and Proposition 4.7.

\section{References}

[1] S. Billey and V. Lakshimibai, Singular loci of Schubert varieties, Progress in Mathematics 182, Birkhäuser, 2000.

[2] M. Brion and P. Polo, Generic singularities of certain Schubert varieties, Math. Z. 231 (1999), no. 2, 301-324.

[3] J. Hong and N. Mok, Analytic continuation of holomorphic maps respecting varieties of minimal rational tangents and applications to rational homogeneous manifolds, J. Differential Geom. 86 (2010), no. 3, 539-567.

[4] _ Characterization of smooth Schubert varieties in rational homogeneous manifolds of Picard number 1, J. Algebraic Geom. 22 (2013), no. 2, 333-362.

[5] J. Hong and K.-D. Park, Characterization of standard embeddings between rational homogeneous manifolds of Picard number 1, Int. Math. Res. Not. 2011 (2011), no. 10, 2351-2373.

[6] J.-M. Hwang and N. Mok, Rigidity of irreducible Hermitian symmetric spaces of the compact type under Kähler deformation, Invent. Math. 131 (1998), no. 2, 393-418.

[7] Deformation rigidity of the 20-dimensional $F_{4}$-homogeneous space associated to a short root, In: Algebraic transformation groups and algebraic varieties, pp. 37-58, Encyclopedia Math. Sci., 132, Springer-Verlag, Berlin, 2004.

[8] _ Prolongations of infinitesimal linear automorphisms of projective varieties and rigidity of rational homogeneous spaces of Picard number 1 under Kähler deformation, Invent. Math. 160 (2005), no. 3, 591-645.

[9] V. Lakshmibai and J. Weyman, Multiplicities of points on a Schubert variety in a minuscule $G / P$, Adv. Math. 84 (1990), no. 2, 179-208.

[10] I. A. Mihai, Odd symplectic manifolds, Transformation Groups 12 (2007), no. 3, 573599. 
Department of Mathematical Sciences

Seoul National University

SEOUL 151-742, KoreA

E-mail address: jhhong00@snu.ac.kr 